Vaucanson et les machines qu'il a imaginées et construites - machines merveilleuses ou utilitaires - illustrent deux faces des rapports hommes/machines : d'une part, la fascination pour la maîtrise des techniques qui miment le réel et allègent la peine ; d'autre part la crainte de la dépossession et de la déqualification des sociétés humaines.

Au-delà des réalités mécaniques, ce sont des usages nouveaux et des représentations du rapport aux objets, à la technique et à la science qui ont été initiés. Ces inventions et celles qui s'inscrivent dans la lignée de la relation mécanisation/substitution des gestes humains posent une nouvelle manière de concevoir l'organisation des sociétés et un autre type de regard sur l'homme et ses capacités. Il s'agit de nouer ce lien entre l'homme et la machine dans toutes ses dimensions. Celles-ci vont des transformations concrètes des pratiques professionnelles et domestiques aux modifications des mentalités qu'elles suggèrent. Doit être aussi considérée la manière dont ces machines, mais aussi leurs concepteurs et promoteurs, créent momentanément ou durablement du mythe, que celui-ci soit positif ou négatif. Comment se fait-il que certaines d'entre elles et certains d'entre eux soient magnifiés ou diabolisés alors que d'autres apparaissent comme banales et tombent dans l'oubli ? Comment analyser les réactions des hommes face à l'imposition des machines dans leur environnement professionnel ou quotidien ?

Ces journées ne prétendent pas faire une synthèse sur ces questions mais bien plus, à partir d'un certain nombre de cas ou d'exemples concrets, permettre de questionner ces différents aspects autour de trois thématiques générales, sans exclusive a priori de champ chronologique ou spatial :

- le rapport au travail et la mutation des pratiques

- le quotidien et l'insertion dans le système des objets

- la construction des réactions (technophile vs technophobe)

Organisation :

Anne-Marie Granet-Abisset, UMR LARHRA, Université Pierre Mendès-France

Anne Dalmasso, UMR LARHRA, Université Pierre Mendès-France

Chantal Spillemaecker, Musée dauphinois, Grenoble

Lieux :

Jeudi 22 janvier 2009 : Musée dauphinois - 30 rue Maurice Gignoux - Grenoble

Renseignements : 0457588901 http://www.musee-dauphinois.fr

Vendredi 23 janvier 2009 : MSH-Alpes - 1221 av. centrale - Domaine universitaire Grenoble - Arrêt tram : Bibliothèques universitaires

Renseignements : 0476827300 - http://www.msh-alpes.fr/Acces/index.htm

\title{
des Machines et des Hommes
}

\section{Mythes et contre-mythes}

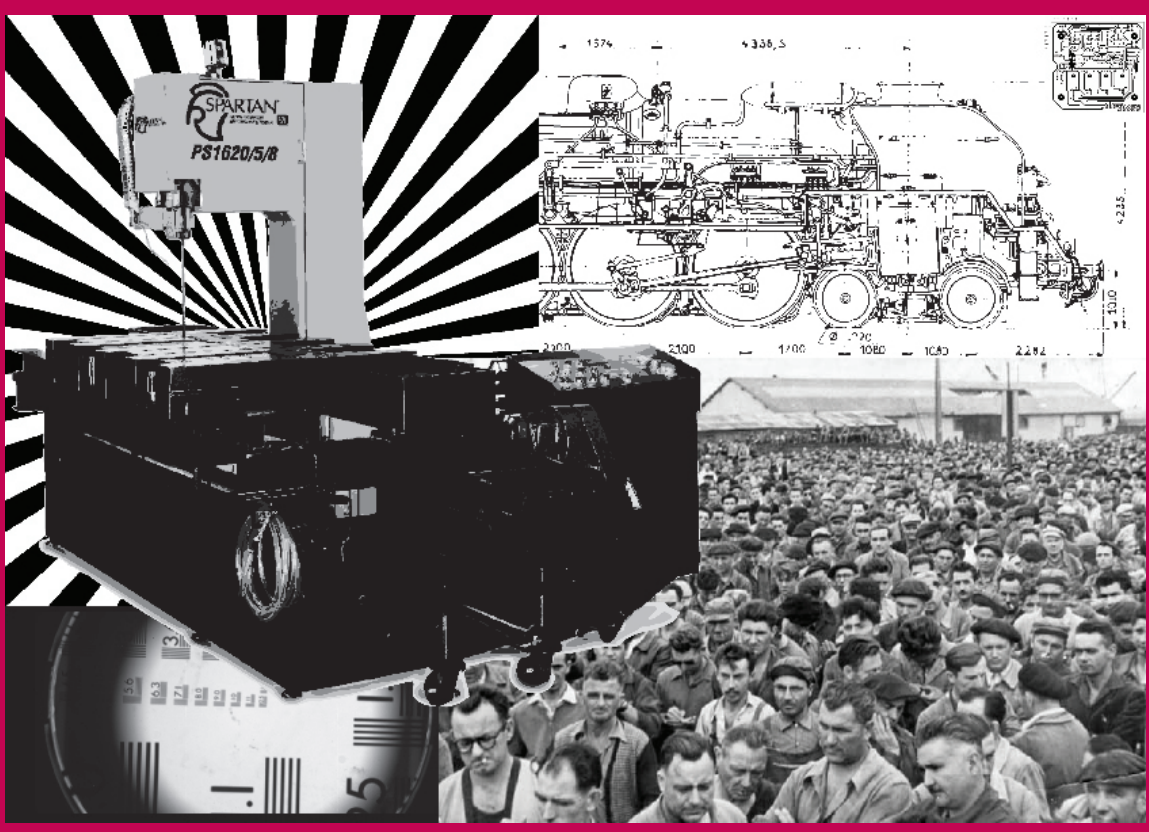

Journées d'études

Jeudi 22 - Vendredi 23 janvier 2009

Photo : @ Nicolas Krautberger, LARHRA

Grenoble 


\section{Jeudi 22 janvier 2009}

Musée dauphinois

09h00-09h30 • Ouverture des journées

Jean-Claude DUCLOS, Directeur du Musée dauphinois, Bernard ROUKHOMOVSKY, président du comité d'organisation du tricentenaire Vaucanson

\section{9h45-10h35 - Introduction}

Anne DALMASSO, Maître de conférences, LARHRA, UPMF

Anne-Marie GRANET-ABISSET, Professeur, LARHRA, UPMF

10h00-12h30

Le rapport au travail et la mutation des pratiques :

gestes, compétences, hiérarchies : le cas du textile

Présidence : Corinne MAITTE, professeur, Université Marne la Vallée

- Les difficultés des débuts de la mécanisation dans le textile

(coton et indiennage, laine et lin) entre 1780 et 1830

Serge CHASSAGNE, Professeur émérite, LARHRA, Université Lyon 2

- Les moulinières et leurs moulins : un "discours en creux "

Florence CHARPIGNY, Ingénieur d'études, CNRS, LARHRA, Lyon

- Pourquoi mécaniser ? le cas de la Schappe à Briançon

Franck DELLION, Docteur en histoire, LARHRA, Lyon

14h00-17h30 L'inventeur et ses machines

Présidence : Serge CHASSAGNE , Professeur émérite, Université Lyon 2

- Vaucanson et les interfaces

Frédéric KAPLAN, Chercheur associé, Ecole polytechnique fédérale de Lausanne

- Aristide Bergès, Machines et mythes d'hier à aujourd'hui

Louis ANDRÉ, Maître de conférences, Université Rennes 2

- L'inventeur et ses automates : pratiques actuelles et perspective historique

Jean-Arcady MEYER, Directeur de recherches CNRS, AnimatLab.

$$
\text { Laboratoire d'Informatique de Paris VI }
$$

- Le minitel, invention et obsolescence

Philippe DENOYELLE, Association Aconit, Grenoble

\section{Vendredi 23 janvier 2009}

MSH-Alpes

09h15-09h30 • Ouverture - Bernard BOUHET, Directeur de la MSH-Alpes

9h30-12 h45 Machines emblématiques, de l'usage au quotidien à la mise en musée

Présidence : Chantal SPILLEMAECKER, conservateur en chef, Musée Dauphinois

- Extériorité et intériorité de l'automobile : la révolution de l'agrandissement de soi Mathieu FLONNEAU, Maître de conférences, Université Paris 1

- Le petit écran : dieu ou démon ? Les rapports amour/haine entre les téléspectateurs français et leur téléviseur

Isabelle GAILLARD, Maître de conférences, LARHRA, UPMF

- La preuve par l'exemple : la mécanique de la modernisation à travers les démonstrations de machines agricoles

Sylvain BRUNIER, Doctorant, LARHRA, UPMF, Grenoble

- La machine, de l'usine au musée : quelles logiques ?

Nadine BESSE, Directrice, Musée d'Art et d'Industrie de Saint-Étienne

- La machine au musée : quelle pérennité ?

Brigitte RIBOREAU, Directrice, Musée de Bourgoin-Jallieu

14h30-17h30 Amour, haine et crainte, autour du couple technophile/technophobe Présidence et conclusion : Anne Françoise Garçon, Université Paris 1

- Jacques Vaucanson et les paradoxes de l'innovation

Bruno JACOMY, Directeur adjoint, Musée des Confluences, Lyon

- Les briseurs de machines, genèses et usages d'un mythe François JARRIGE, ATER, Université du Maine

- Le barrage comme « modèle, machine, merveille » : de l'idéalisation au rejet Anne DALMASSO, Maître de conférences, LARHRA, UPMF

- Usage des TICs, Paradoxes, Enjeux identitaires et Nouveau régime de savoir Philippe MALLEIN, Ingénieur de recherche, CNRS, Minatech Ideaslab, Grenoble 